\title{
Desenvolvimento e validação de método analítico para análise de 2,4-D, 2,4-DCP e 2,4,5-T para monitoramento em água de abastecimento público
}

\author{
Development and validation of analytical method for analysis of \\ 2,4-D, 2,4-DCP and 2,4,5-T for monitoring of public water supply
}

\section{Edumar Ramos Cabral Coelho** ${ }^{\oplus}$, Waldiléia Pereira Leal' ${ }^{\circledR}$, Karoline Barros de Souza', Andréia do Rozário ${ }^{2}\left(\mathbb{0}\right.$, Paulo Wagnner Pereira Antunes ${ }^{1}[$}

$\square$

\section{RESUMO}

Oácido 2,4-diclorofenoxiacético (2,4-D) é o segundo herbicida mais consumido no Brasil e seus efeitos na contaminação dos recursos hídricos e consequente risco sanitário para abastecimento público são conhecidos. O 2,4-D e seu principal produto de degradação, o 2,4-diclorofenol (2,4-DCP), apresentam potencial de desregulação endócrina, e o ácido 2,4,5-triclorofenoxiacético (2,4,5-T) é tóxico e persistente no meio ambiente. A inexistência de uma metodologia para quantificação simultânea desses compostos motivou o desenvolvimento e a validação do método de extração e concentração de amostras ambientais e a quantificação em cromatografia líquida de alta eficiência com detector de arranjo de diodos (CLAE-DAD) para análise de 2,4-D, 2,4-DCP e2,4,5-T emágua filtrada produzida em estação de tratamento deágua e água de manancial superficial. Os resultados de extração em fase sólida e a concentração da amostra demonstraram recuperação de 89 a 119\%, e desvio padrão entre 0,9 e 11,4\%. Para os ensaios de identificação e quantificação, os limites de detecção variaram entre 0,17 e 0,51 $\mu \mathrm{g} \cdot \mathrm{L}^{-1} \mathrm{e}$ limite de quantificação de $1,0 \mu \mathrm{g} . \mathrm{L}^{-1}$. Os resultados mostraram que é possível empregar esse método na quantificação do 2,4-D, do 2,4-DCP e do 2,4,5-T em monitoramento ambiental e em sistemas de abastecimento de água atendendo às legislações brasileiras. Palavras-chave: cromatografia líquida de alta eficiência; ácido 24-diclorofenoxiacético; 2,4-diclorofenol; ácido 2,4,5-triclorofenoxiacético; extração em fase sólida.

\begin{abstract}
The 2,4-dichlorophenoxyacetic acid (2,4-D) is the second most consumed herbicide in Brazil and their effects in the contamination of the water resources and consequent sanitary risk for public supply are known. The 2,4-D and its main degradation product, 2,4-dichlorophenol (2,4-DCP), have the potential for endocrine disruption and 2,4,5-trichlorophenoxyacetic acid (2,4,5-T) is toxic and persistent in the environment. The absence of a methodology that simultaneously determines these compounds led to the development and validation of the solid phase extraction method, environmental samples concentrations, identification and quantification in HPLC-DAD for 2,4-D, 2,4-DCP and 2,4,5-T analysisin filtered water, produced at water treatment plants, and surface water. The extraction essay and samples concentration showed recovery from 85 to 119\%, standard deviation between 0.9 and $11.4 \%$. For identification and quantification essays, detection limits ranged between 0.17 and $0.51 \mu \mathrm{g} . \mathrm{L}^{-1}$ and the quantification limit was $1.0 \mu \mathrm{g} \mathrm{L}^{\mathrm{L}^{-1}}$. The results showed that it is possible to employ this method for the quantification of 2,4-D, 2,4-DCP and 2,4,5-T in environmental monitoring and in water supply systems taking account of Brazilian regulatory agencies
\end{abstract}

Keywords: high performance liquid chromatography; 2,4-dichlorophenoxyacetic acid; 2,4-dichlorophenol; 2,4,5-trichlorophenoxyacetic acid; solid phase extraction.

\section{INTRODUÇÃO}

O ácido 2,4-diclorofenoxiacético (2,4-D) é um herbicida popular, seletivo e sistêmico, bem como um regulador de crescimento, que é amplamente utilizado em todo o mundo em plantas de folhas largas (NJOKU et al., 2015). Os efeitos potenciais de toxicidade do 2,4-D e seus produtos de transformação, tal como o 2,4-diclorofenol (2,4-DCP), têm sido documentados por vários pesquisadores, além do 2,4-DCP ter sido classificado pela União Europeia (UE) como uma substância de desregulação endócrina ou potencial de desregulação endócrina comprovada (CCE, 2001; XI; MALLAVARAPU; NAIDU, 2010). No Brasil, a Agência Nacional de Vigilância Sanitária (Anvisa) classificou o 2,4-D como extremamente tóxico (Classe I) para a saúde, e 
estudos recentes da Agência Internacional de Pesquisas do Câncer (IARC), com a Organização Mundial de Saúde (OMS), classificaram o 2,4-D como possivelmente cancerígeno a humanos (LOOMIS et al., 2015). Além disso, outras pesquisas têm avaliado a associação entre o 2,4-D e o linfoma não-Hodgkin (GOODMAN; LOFTUS; ZU, 2017; SMITH et al., 2017). Já o ácido 2,4,5-triclorofenoxiacético (2,4,5-T) foi utilizado mundialmente em grande escala na agricultura para controlar o crescimento de ervas daninhas de folhas largas e em aplicações pós-emergência. Durante a Guerra do Vietnã, o 2,4,5-T, associado ao 2,4-D e ao pentaclorofenol, deu origem ao famoso "agente laranja", que foi utilizado pela força aérea americana como agente desfolhante (TU; HURD; RANDALL, 2001). Ao ser comparado com o 2,4-D, possui menor biodegradabilidade e maior resistência ao metabolismo microbiano, podendo ser detectado em águas superficiais e subterrâneas, não apenas durante a sua aplicação, mas também após um longo período (WANG \& CHU, 2011).

As fórmulas estruturais dos compostos 2,4-D, 2,4-DCP e 2,4,5-T são apresentadas na Figura 1.

O uso indiscriminado de ácidos fenoxiacéticos clorados, como herbicidas seletivos, tem levado ao aumento da poluição das águas, do solo e dos alimentos, além de terem sido identificados como alérgenos do sistema respiratório. Em razão da sua alta toxicidade em mamíferos, em organismos aquáticos e da sua persistência ambiental em longo prazo, as legislações colocam limites rigorosos sobre a presença desses agrotóxicos como resíduos, sujeito às análises de traços (BAGGIANI et al., 2001; FUKUYAMA et al., 2009).

Pesquisas direcionadas ao entendimento do destino ambiental dessas substâncias e dos efeitos adversos causados a organismos não alvos têm

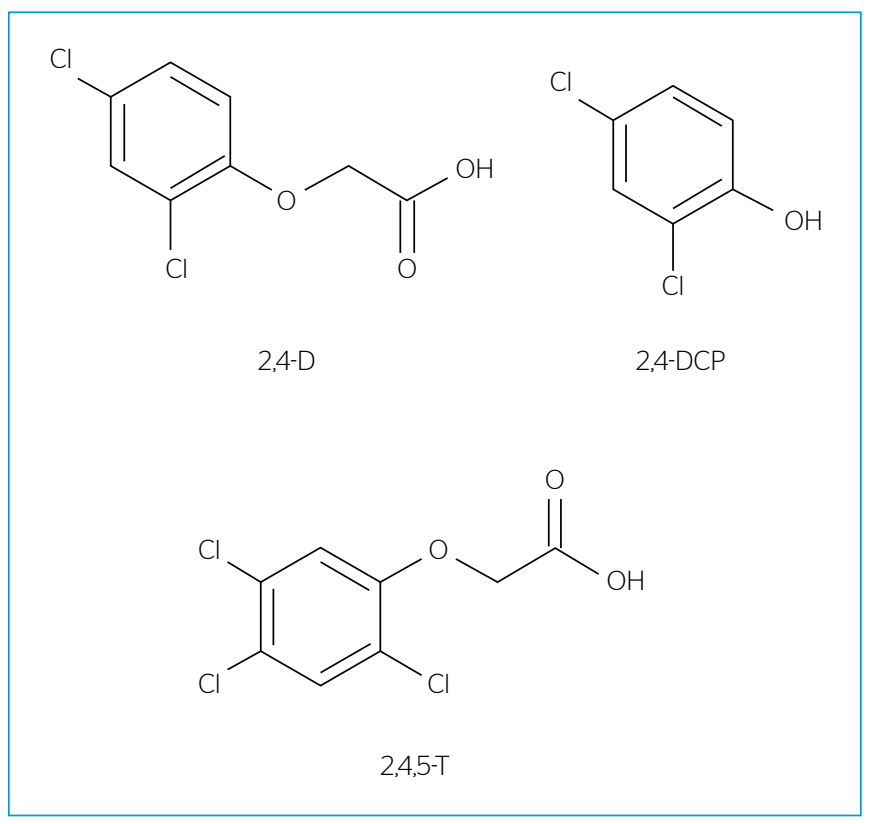

Figura 1 - Fórmula estrutural do 2,4-D, do 2,4-DCP e do 2,4,5-T. evidenciado a ocorrência de resíduos de agrotóxicos no meio ambiente (SALVAGNI; TERNUS; FUENTEFRIA, 2011), em águas superficiais e em águas subterrâneas (AZEVEDO; GERCHON; REIS, 2004; DE ARMAS et al., 2007; RIBEIRO et al., 2013). A presença dos ácidos fenoxiacéticos nos corpos hídricos se dá em razão da sua solubilidade em água, do seu baixo valor de $\mathrm{pKa}$, da sua baixa biodegradabilidade e por serem fracamente retidos pela maioria dos solos devido seu baixo coeficiente de adsorção no solo (NJOKU \& HAMEED, 2011; XI; MALLAVARAPU; NAIDU, 2010; SOUZA et al., 2017). Resultados indicativos da presença de 2,4-D em mananciais apresentaram valores abaixo de $30 \mu \mathrm{g} . \mathrm{L}^{-1}$, porém foi detectado em até $26 \%$ do total das amostras analisadas (MARCHESAN et al., 2010; MCKNIGHT et al., 2015; PRIMEL et al., 2005).

Estudos também evidenciaram a presença de agrotóxicos em águas utilizadas para consumo humano (DORES et al., 2006; NAM et al., 2014; RISSATO et al., 2004), sendo que a contaminação de mananciais por agrotóxicos apresenta risco sanitário à saúde e devem ser monitorados também em sistemas de abastecimento de água. A Portaria do Ministério da Saúde (MS) no 2.914/2011 estabelece o valor máximo permitido (VMP) de $30 \mu \mathrm{g} . \mathrm{L}^{-1}$ para a soma do $2,4-\mathrm{D}$ e do $2,4,5-\mathrm{T}$, não fazendo referência ao 2,4-DCP (BRASIL, 2011).

A separação e a quantificação desses herbicidas podem ser realizadas por técnicas analíticas de cromatografia líquida de alta eficiência (CLAE). Os métodos de análise do 2,4-D e do 2,4-DCP por CLAE costumam ser mais simples e rápidos, se comparados com sua determinação por cromatografia gasosa (CG) (AULAKH et al., 2005). No entanto, apesar de o 2,4,5-T ter sido incluído na legislação de potabilidade brasileira, devido sua persistência ambiental, na literatura não foram encontradas referências acerca da determinação simultânea desses compostos por meio de métodos cromatográficos.

Portanto, o objetivo deste estudo foi desenvolver e validar um método de separação e quantificação de 2,4-D, 2,4-DCP e 2,4,5-T em água filtrada e em água de manancial superficial utilizando a CLAE.

\section{METODOLOGIA}

\section{Reagentes}

Os reagentes utilizados foram acetonitrila (ACN) grau CLAE (J.T. Baker), padrões analíticos de 2,4-D, 2,4-DCP e 2,4,5-T grau CLAE com pureza $\geq 99 \%$ e formiato de amônio P.A (Sigma, Aldrich) com pureza $\geq 98 \%$. A água ultrapura foi purificada no sistema de ultrapurificação (Millipore) e acidificada com ácido fosfórico P.A (Synth), com pureza de $85 \%$.

\section{Análises cromatográficas}

As análises para detecção do 2,4-D, do 2,4-DCP e do 2,4,5-T foram realizadas em um sistema de cromatografia (Shimadzu), modelo CBM-20A, equipado com módulo desgaseificador de solventes (DGU-20AS), bomba 
quaternária de gradiente (LC-20AT), injetor automático de amostras (SIL-20AHT), detector de arranjo de diodos (DAD) (SPD-M20A) e forno de coluna (CTO-20A). A coluna cromatográfica utilizada neste estudo foi a coluna analítica XTerra MS C18 $(150 \times 4,6 \mathrm{~mm}, 3,5 \mu \mathrm{m})$.

A fase móvel (FM) foi composta por uma solução de ACN e formiato de amônio 10,0 mM, acidificado com 0,10\% de ácido fosfórico (ACN: $\mathrm{NH}_{4} \mathrm{COOH} 47: 53 \mathrm{v} / \mathrm{v}$ ), com eluição isocrática, no fluxo de 1,2 mL. $\min ^{-1}$. O volume de injeção de amostras para as análises foi de $50,0 \mu \mathrm{L}$, com temperatura de acondicionamento da coluna de $30^{\circ} \mathrm{C}$, e identificação e quantificação dos analitos em comprimentos de onda de 200 e $206 \mathrm{~nm}$.

Todas as soluções preparadas para compor as FMs foram previamente filtradas em membrana de PTFE 0,45 $\mu \mathrm{m}$ (Millipore) e desgaseificadas em banho de ultrassom (Limpsonic), por 15 minutos, sob vácuo.

\section{Coleta e preparo de amostras}

As soluções estoque dos analitos, 2,4-D, 2,4-DCP e 2,4,5-T, foram preparadas individualmente em ACN na concentração 0,10 g.L.-1 . A partir dessas soluções, foram preparadas as soluções padrão em água ultrapura para a validação do método.

Em amostras naturais, faz-se necessário a concentração e o isolamento do analito, além de eliminar possíveis interferentes da matriz. No presente trabalho, foi utilizada a extração em fase sólida (EFS).

$\mathrm{Na}$ EFS, volumes de $50 \mathrm{~mL}$ de cada amostra acidificada com ácido fosfórico $(0,10 \%)$ foram passados por um sistema a vácuo para a concentração das amostras. As amostras foram percoladas em cartuchos $\mathrm{C}_{18}$ (500 mg) da Agilent previamente condicionados com $10 \mathrm{~mL}$ de ACN e $10 \mathrm{~mL}$ de água ultrapura. Posteriormente, os cartuchos foram lavados com $5 \mathrm{~mL}$ de água ultrapura e o analito foi eluído em $1 \mathrm{~mL}$ de ACN. O eluído foi acrescido de $1 \mathrm{~mL}$ de água ultrapura e analisado em CLAE.

As amostras de água bruta e de água filtrada foram coletadas na estação de tratamento de água (ETA) de Carapina na Serra, Espírito Santo, cujo manancial abastecedor é o rio Santa Maria da Vitória, responsável pelo abastecimento público da região metropolitana de Vitória, Espírito Santo. As coletas foram realizadas semanalmente durante o mês de novembro de 2012. A caracterização físico-química foi realizada diariamente e é apresentada na Tabela 1. As amostras eram acondicionadas em bombonas de plástico de 20 L e encaminhadas ao Laboratório de Saneamento da Universidade Federal do Espírito Santo (UFES) para análise e permaneciam em ambiente refrigerado e sem exposição direta à luz. As amostras coletadas para realização da validação da metodologia eram mantidas em frascos âmbar a $4^{\circ} \mathrm{C}$ e filtradas em filtro de seringa PTFE Teflon 0,2 $\mu \mathrm{m}$ (M.Nagel) antes das análises cromatográficas.

\section{Validação do método}

Os parâmetros avaliados para a validação do método EFS-CLAE-DAD foram: seletividade, linearidade, limite de detecção (LD), limite de quantificação (LQ), recuperação, precisão, exatidão e robustez. Eles foram determinados de acordo com a Resolução nº 899/2003 da Anvisa e recomendações da Association of Official Analytical Chemistry para análise de resíduos (ANVISA, 2003; AOAC, 1998; WOOD, 1999). O método foi desenvolvido por meio da otimização dos parâmetros seguindo as recomendações de Lanças (2012), e a etapa de EFS foi adaptada do método proposto por Faria (2004). Os dados foram processados utilizando o software LCSolutions (versão 2.1).

\section{RESULTADOS E DISCUSSÃO}

\section{Validação das condições cromatográficas}

Para a seleção do comprimento de onda foram analisados os espectros de absorção na região do ultravioleta (UV) na faixa de 190 a $300 \mathrm{~nm}$. No comprimento de onda de $200 \mathrm{~nm}$, obteve-se máxima absorção para o 2,4-D e o 2,4-DCP, e em $206 \mathrm{~nm}$ foi obtida a máxima absorção de 2,4,5-T, conforme apresentado na Figura 2.

Foram testadas diferentes FMs com eluição isocrática (FM1, FM2, FM3, FM4) antes de chegar na FM do método. A primeira FM testada (FM1) foi a mistura de metanol e $\mathrm{H}_{2} \mathrm{O}(50: 50 \mathrm{v} / \mathrm{v})$ acidificada com $\mathrm{H}_{3} \mathrm{PO}_{4}(0,10 \%)$ (FARIA, 2004), mas o resultado não foi eficiente, mesmo variando a concentração de metanol e o pH da solução. Para chegar à FM2 foram realizados vários testes variando a concentração de $\mathrm{ACN}$, e o melhor resultado foi obtido utilizando $\mathrm{ACN} \mathrm{e}_{2} \mathrm{O}(42: 58 \mathrm{v} / \mathrm{v})$ acidificada com $\mathrm{H}_{3} \mathrm{PO}_{4}(0,10 \%)$. Visando à otimização do método, foi testado o uso do tampão formiato de amônio na FM3, utilizando a mistura de metanol e $10 \mathrm{mM}$ de formiato de amônio (50:50 v/v) acidificada com $\mathrm{H}_{3} \mathrm{PO}_{4}(0,10 \%)$; e na FM4, a FM era constituída de ACN e $10 \mathrm{mM}$ de formiato de amônio (42:58 v/v) acidificada com $\mathrm{H}_{3} \mathrm{PO}_{4}(0,10 \%)$.

Tabela 1 - Caracterização das amostras de água.

\begin{tabular}{|c|c|c|}
\hline \multirow{2}{*}{ Parâmetros (unidades) } & $\begin{array}{l}\text { Água do rio Santa } \\
\text { Maria da Vitória }\end{array}$ & Água filtrada \\
\hline & mínimo - máximo & mínimo - máximo \\
\hline pH (adimensional) & $7,3-7,6$ & $6,3-7,1$ \\
\hline Temperatura $\left({ }^{\circ} \mathrm{C}\right)$ & $21-25$ & $20-23$ \\
\hline Turbidez (uT) & $23,1-37,0$ & $0,19-0,57$ \\
\hline Cor aparente (uH) & $160-244$ & $1-2$ \\
\hline Cor real $(\mathrm{uH})$ & $16-23$ & nd \\
\hline Alcalinidade (mgCaCO$\left.{ }_{3} \mathrm{~L}^{-1}\right)$ & $19-24$ & $8,5-14,0$ \\
\hline Condutividade $\left(\mu \mathrm{S} . \mathrm{cm}^{-2}\right)$ & $50-56$ & $52-54$ \\
\hline Absorbância UV $\left(\mathrm{cm}^{-1}\right)$ & $0,061-0,097$ & $0,018-0,021$ \\
\hline Cloro livre (mg. $\mathrm{L}^{-1}$ ) & - & $0,12-1,05$ \\
\hline COT (mg. $\left.L^{-1}\right)$ & $8,44-8,96$ & $1,28-1,80$ \\
\hline $\operatorname{COD}\left(\mathrm{mg} \mathrm{L}^{-1}\right)$ & $7,10-8,50$ & - \\
\hline
\end{tabular}

UV: ultravioleta; COT: carbono orgânico total; COD: carbono orgânico dissolvido. 
Foi avaliada também a variação na temperatura da coluna, o volume de injeção e o fluxo.

A FM otimizada foi composta por uma solução de ACN e formiato de amônio 10,0 $\mathrm{mM}$ acidificado com $0,10 \%$ de ácido fosfórico, ACN:10 mmol.L $\mathrm{L}^{-1} \mathrm{NH}_{4} \mathrm{COOH}(47: 53 \mathrm{v} / \mathrm{v})$, empregando eluição isocrática com volume de injeção de $50,0 \mu \mathrm{L}$ da amostra com fluxo de $1,2 \mathrm{~mL} \cdot \mathrm{min}^{-1}$ na temperatura de $30^{\circ} \mathrm{C}$.

Os tempos de retenção $\left(t_{R}\right)$ foram encontrados injetando soluções padrões $(\mathrm{n}=7)$ na concentração de $100 \mu \mathrm{g} . \mathrm{L}^{-1}$, com os três analitos e com soluções individuais destes, no mesmo dia e em dias diferentes, com um coeficiente de variação $(\mathrm{CV})$ menor que $5 \%$. $\mathrm{O}_{\mathrm{R}}$ obtido foi de 3,92 ; 4,73 e 5,49 minutos para 2,4-D, 2,4-DCP e 2,4,5-T, respectivamente.

\section{Seletividade}

A seletividade de um método instrumental de separação é a capacidade de avaliar, de forma inequívoca, o analito de interesse na presença de componentes que podem estar presentes na amostra de água natural, como impurezas. A seletividade do método foi determinada analisando a possível presença de componentes que pudessem eluir no $t_{R}$ dos analitos de interesse, interferindo nas análises. Dessa forma, foram analisadas três diferentes amostras do branco da matriz $(n=3)$ e da matriz fortificada para verificar a possível presença de interferentes. O resultado foi comparado à amostra padrão e está apresentado na Figura 3. Na Figura 3A, é apresentado o cromatograma de uma amostra do branco da matriz-água do rio Santa Maria da Vitória, que demonstra a ausência de interferentes nos $t_{R}$ dos analitos, indicando a seletividade da metodologia proposta. Na Figura 3B, tem-se o cromatograma obtido com a amostra de água do rio Santa Maria da Vitória fortificada com os três agrotóxicos em estudo; e na Figura 3C, observa-se o cromatograma da amostra padrão com a adição dos três compostos em água ultrapura. Em todas as amostras a concentração dos analitos foi de 100,0 $\mu \mathrm{g} \cdot \mathrm{L}^{-1}$.

\section{Linearidade}

A linearidade do método foi determinada a partir das curvas analíticas obtidas pela injeção de soluções padrões $(n=7)$ em 7 concentrações dos analitos — 20,0;30,0; 50,0;76,0; 100,0; 126,0; e 150,0 $\mu \mathrm{g} . \mathrm{L}^{-1}$ — em água

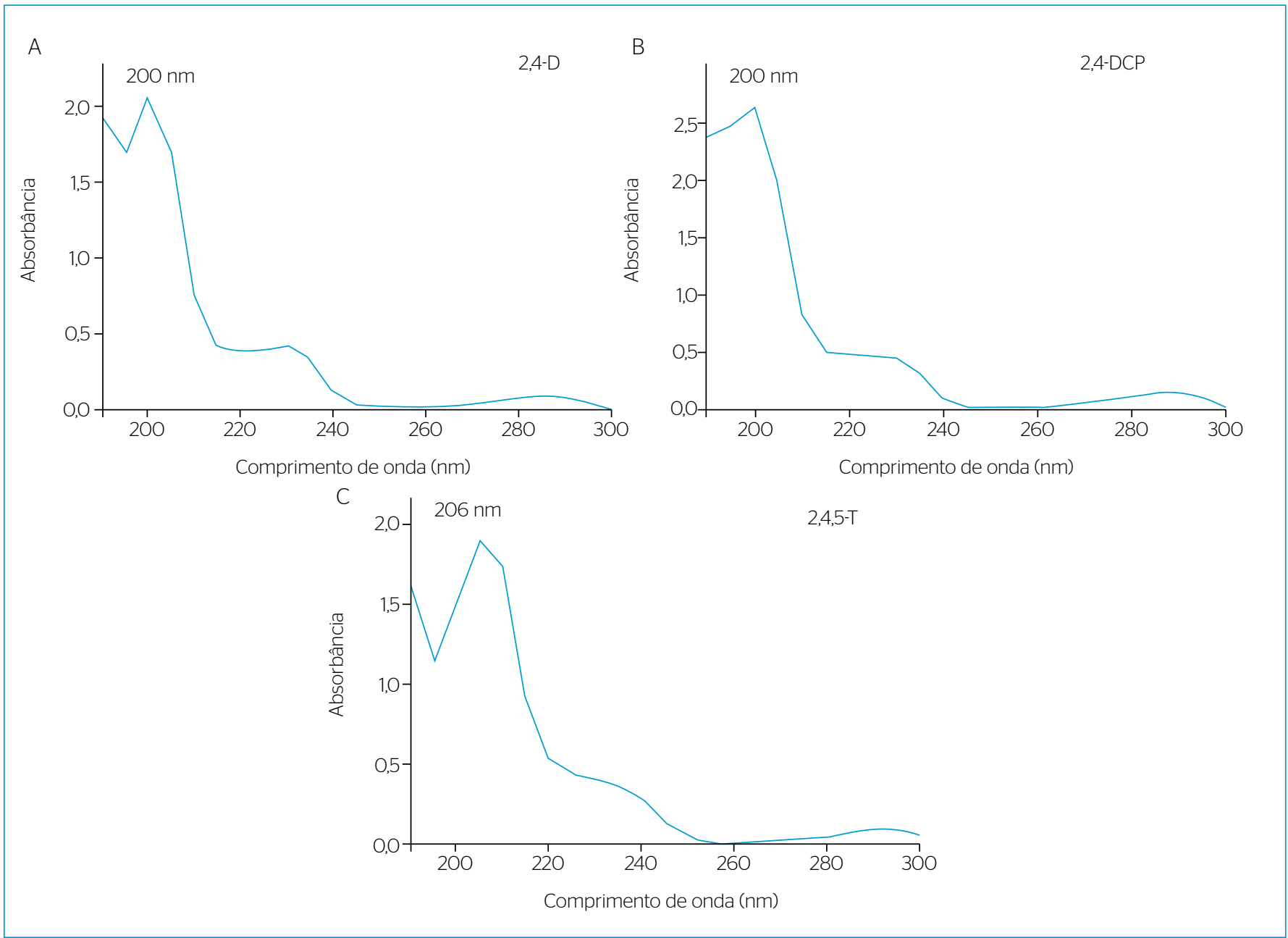

Figura 2 - Perfis cromatográficos de máxima absorção na região do ultravioleta dos analitos (A) 2,4-D; (B) 2,4-DCP e (C) 2,4,5-T determinados por cromatografia líquida de alta eficiência com detector de arranjo de diodos. 
ultrapura. A linearidade da resposta de detecção dos analitos pela técnica cromatográfica foi avaliada pela relação entre a concentração injetada e a área do pico de resposta. Os resultados são apresentados na Tabela 2.

Os resultados demonstraram a capacidade da técnica em correlacionar diretamente os valores da concentração dos analitos na amostra com a área do pico de resposta para todos os analitos avaliados. A resposta linear da técnica foi satisfatória, apresentando coeficientes de correlação (r) superiores a 0,99, estando de acordo com as normas exigidas pela Anvisa (2003).

Valores de coeficientes semelhantes também foram observados em diferentes estudos para determinação de 2,4-D e 2,4-DCP, utilizando técnica de CLAE. Em separações utilizando o sistema de deteç̧ão DAD, observou-se a linearidade no intervalo de concentração de 2,4-D de 40 a $140 \mu \mathrm{g} . \mathrm{L}^{-1}$, com (r) de 0,9984 (D’ARCHIVIO et al., 2007). Utilizando o sistema de detecção UV, observou-se a linearidade no intervalo de

Tabela 2 - Tempo de retenção, comprimento de onda, coeficiente de variação, equação da curva de calibração e coeficiente de correlação.

\begin{tabular}{|c|c|c|c|c|c|c|}
\hline \multirow[t]{2}{*}{ Analitos } & \multirow{2}{*}{$t_{R}$} & \multirow{2}{*}{$\underset{(\mathrm{nm})}{\lambda}$} & \multirow{2}{*}{$\begin{array}{c}\text { Concentração } \\
\left(\mu \mathrm{g} \cdot \mathrm{L}^{-1}\right)\end{array}$} & \multirow{2}{*}{ CV (\%) } & \multicolumn{2}{|c|}{$\begin{array}{l}\text { Dados da curva } \\
\text { de calibração }\end{array}$} \\
\hline & & & & & go & $r$ \\
\hline $2,4-D$ & 3,9 & 200 & 20 a 150 & $1,3-5,1$ & $y=461,69 x-884$ & 0,9989 \\
\hline 2,4-DCP & 4,7 & 200 & 20 a 150 & $1,6-4,1$ & $y=629,06 x-2139$ & 0,9991 \\
\hline $2,4,5-T$ & 5,4 & 206 & 20 a 150 & $1,3-4,2$ & $y=425,05 x-1611$ & 0,9985 \\
\hline
\end{tabular}

$\mathrm{t}_{\mathrm{R}}$ : tempo de retenção; $\lambda$ : comprimento de onda; $\mathrm{CV}$ : coeficiente de variação; $\mathrm{r}$ : coeficiente de correlação.

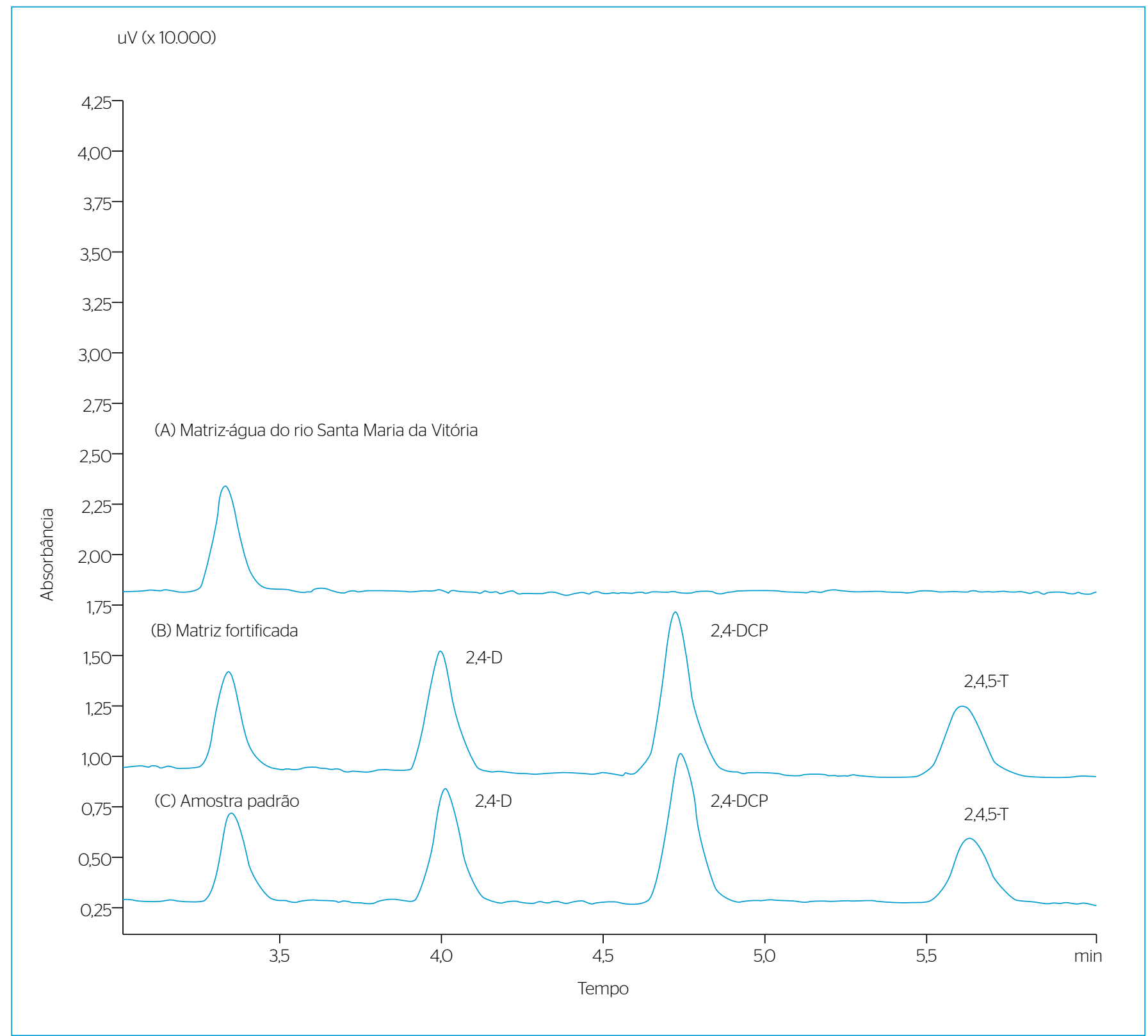

Figura 3 - Perfis cromatográficos (cromatografia líquida de alta eficiência com detector de arranjo de diodos): (A) amostra do branco da matriz-água do rio Santa Maria da Vitória; (B) amostra da água do rio Santa Maria da Vitória fortificada com os analitos (100 $\left.\mu \mathrm{g} . \mathrm{L}^{-1}\right)$; (C) amostra padrão (100 $\left.\mu \mathrm{g} \cdot \mathrm{L}^{-1}\right)$. 
100 a $1.000 \mu \mathrm{g} . \mathrm{L}^{-1}$, com (r) de 0,9989 para análise de 2,4-D (BRONDI \& LANÇAS, 2005). Para a detecção de 2,4-DCP foi observado valor de (r) de 0,9982, para o intervalo de concentração de 2.000 a $10.000 \mu \mathrm{g} . \mathrm{L}^{-1}$ (MACEDO NETO; FROEHNER; MACHADO, 2012), e de 0,9905, para linearidade de 100 a $10.500 \mu \mathrm{g} . \mathrm{L}^{-1}$ (LOPES et al., 2011).

\section{Precisão e exatidão}

Precisão representa a dispersão de resultados entre ensaios independentes e repetidos, de uma mesma amostra, de amostras semelhantes ou padrões, sob condições definidas; e pode ser avaliada pelo CV (RIBANI et al., 2004, ANVISA, 2003). A precisão do método foi avaliada por meio da repetitividade, em ensaios realizados no mesmo dia, das soluções preparadas em triplicata e em três níveis de fortificação 1,0, 3,0 e 5,0 $\mu \mathrm{g} . \mathrm{L}^{-1}$-, expressa por meio do CV, em água natural, conforme recomendações da Anvisa (ANVISA, 2003).

A exatidão do método foi avaliada por meio da EFS e medida pelas respectivas porcentagens de recuperação. $O$ teste de recuperação foi realizado pela adição dos analitos em amostras de água filtrada da ETA e água bruta do rio Santa Maria da Vitória. Volumes de $50 \mathrm{~mL}$ da amostra de água foram coletados e fortificados com os analitos na concentração final de 1,0; 3,0 e 5,0 $\mu \mathrm{g} \cdot \mathrm{L}^{-1}$ com análises em triplicata. Em seguida, foram concentrados em cartuchos de extração tipo $\mathrm{C}_{18} \mathrm{e}$ analisados no sistema cromatográfico. As porcentagens de recuperação obtidas variaram entre 85 e $119 \%$ e a repetibilidade, expressa em CV, foi inferior a $12 \%$, conforme apresentado na Tabela 3.

Esses resultados são considerados satisfatórios para análise de resíduos de agrotóxicos, cujo intervalo de recuperações entre 70 e $120 \%$ com precisão de até 20\% é aceitável (AOAC, 1998; RIBANI et al., 2004; WOOD, 1999). Estudos que avaliaram a recuperação de 2,4-D apresentaram valores que variaram entre 73 e 111\% (MACEDO NETO; FROEHNER; MACHADO, 2012; LOPES et al., 2011), com CV de até $11 \%$ (BRONDI \& LANÇAS, 2005). Para o 2,4-DCP foi encontrado valores entre 73 e 88\% de recuperação, com CV de 5\% (LOPES et al., 2011).

As porcentagens de recuperação obtidas demonstraram que o método está de acordo com o estabelecido pela Anvisa (ANVISA, 2003), que determina que as porcentagens de recuperação próximas de $100 \%$ são desejáveis, porém se admite valores menores, desde que a recuperação seja precisa e exata.

\section{Limite de detecção e limite de quantificação}

O LD e o LQ do método, EFS-CLAE-DAD, foram determinados a partir de soluções nas concentrações de 1,0; 3,0 e 5,0 $\mu \mathrm{g} . \mathrm{L}^{-1}$ dos analitos de estudo. O LD foi calculado a partir dos parâmetros de três curvas de calibração construídas para cada nível de concentração. O LQ foi atribuído às menores concentrações dos analitos presentes nas soluções que puderam ser determinados com precisão e exatidão aceitáveis sob as condições experimentais estabelecidas, com CV dos picos medidos, inferiores a 12\% (ANVISA, 2003). Os valores de LD variam entre 0,17 e $0,51 \mu \mathrm{g} . \mathrm{L}^{-1}$ e o LQ obtido foi de 1,0 $\mu$ g.. $\mathrm{L}^{-1}$, o que pode ser observado na Tabela 4.

A metodologia analítica para separação de agrotóxicos utilizando a técnica de CLAE com detector UV apresentou LD de 25,0 $\mu$ g. $\mathrm{L}^{-1} \mathrm{e}$ LQ de 85,0 $\mu$ g.L.-1 para o 2,4-D (BRONDI \& LANÇAS, 2005). O sistema de separação com o uso do DAD apresentou valores de LD de 0,02 $\mu$ g. $\mathrm{L}^{-1}$ e de LQ de 0,07 $\mu \mathrm{g} . \mathrm{L}^{-1}$ para o herbicida 2,4-D (D’ARCHIVIO et al., 2007). Para o 2,4-DCP, os valores encontrados foram de 22,0 e 92,6 $\mu \mathrm{g} . \mathrm{L}^{-1}$ para LD e LQ, respectivamente (OPEOLU; FATOKI; ODENDAAL, 2010). LD de 0,072 $\mu$ g. $\mathrm{L}^{-1}$ e LQ de $0,22 \mu \mathrm{g} . \mathrm{L}^{-1}$ para o 2,4-D e LD de 0,083 $\mu \mathrm{g}$. $\mathrm{L}^{-1}$ e LQ de $0,25 \mu \mathrm{g}$. $\mathrm{L}^{-1}$ para o 2,4-DCP também foram apresentados quando da utilização de CLAE/UV (LOPES et al., 2011). Os valores de LD e LQ apresentados na Tabela 4 foram considerados satisfatórios para análise de resíduos de agrotóxicos em amostras de água natural, atendendo aos limites determinados pela legislação brasileira para presença de 2,4-D+2,4,5-T (30 $\left.\mu \mathrm{g} . \mathrm{L}^{-1}\right)$ em amostras de águas para abastecimento público.

\section{Robustez}

A robustez de um método mede a sensibilidade apresentada com pequenas variações dos parâmetros. Diz-se que um método é robusto

Tabela 3 - Exatidão (\% recuperação) e repetibilidade (\% CV) do método analítico em amostras de água filtrada e água bruta do rio Santa Maria da Vitória.

\begin{tabular}{|c|c|c|c|c|c|}
\hline \multirow{2}{*}{ Analitos } & \multirow{2}{*}{$\begin{array}{l}\text { Concentração } \\
\left(\mu \mathrm{g} \cdot \mathrm{L}^{-1}\right)\end{array}$} & \multicolumn{2}{|c|}{ Água filtrada } & \multicolumn{2}{|c|}{$\begin{array}{l}\text { Agua do rio Santa } \\
\text { Maria da Vitória }\end{array}$} \\
\hline & & $\begin{array}{c}\text { Recuperação } \\
\text { (\%) }\end{array}$ & CV (\%) & $\begin{array}{c}\text { Recuperação } \\
\text { (\%) }\end{array}$ & CV (\%) \\
\hline \multirow{3}{*}{$2,4-\mathrm{D}$} & 1,0 & 85 & 4,3 & 97 & 3,3 \\
\hline & 3,0 & 98 & 8,1 & 105 & 7,0 \\
\hline & 5,0 & 101 & 0,9 & 102 & 3,4 \\
\hline \multirow{3}{*}{ 2,4-DCP } & 1,0 & 93 & 5,4 & 119 & 4,6 \\
\hline & 3,0 & 88 & 1,5 & 110 & 3,6 \\
\hline & 5,0 & 110 & 2,1 & 108 & 2,4 \\
\hline \multirow{3}{*}{ 2,4,5-Т } & 1,0 & 110 & 5,8 & 109 & 1,4 \\
\hline & 3,0 & 101 & 2,5 & 114 & 11,4 \\
\hline & 5,0 & 109 & 3,0 & 107 & 2,4 \\
\hline
\end{tabular}

$\mathrm{CV}$ : coeficiente de variação.

Tabela 4 - Limite de detecção e limite de quantificação dos analitos.

\begin{tabular}{l|c|c|c|c}
\multirow{2}{*}{ Analitos } & LD $\left(\mu \mathrm{g} . \mathrm{L}^{-1}\right)$ & $\mathrm{LQ}\left(\mu \mathrm{g} . \mathrm{L}^{-1}\right)$ & $\mathrm{LD}\left(\mu \mathrm{g} . \mathrm{L}^{-1}\right)$ & $\mathrm{LQ}\left(\mu \mathrm{g} \cdot \mathrm{L}^{-1}\right)$ \\
\cline { 2 - 5 } & \multicolumn{2}{|c|}{ Água filtrada } & \multicolumn{2}{|c}{$\begin{array}{c}\text { Água do rio } \\
\text { Santa Maria da Vitória }\end{array}$} \\
\hline 2,4-D & 0,17 & 1,00 & 0,42 & 1,00 \\
\hline 2,4-DCP & 0,28 & 1,00 & 0,20 & 1,00 \\
\hline 2,4,5-T & 0,28 & 1,00 & 0,51 & 1,00 \\
\hline
\end{tabular}

LD: limite de detecção; LQ: limite de quantificação. 
quando não é afetado por uma modificação pequena e deliberada em seus parâmetros. Em CLAE, a robustez pode ser avaliada, por exemplo, pela variação de parâmetros como a concentração do solvente orgânico, o pH e a temperatura (RIBANI et al., 2004). A robustez do método foi avaliada por meio dos resultados obtidos de pequenas variações nas temperaturas de acondicionamento da coluna $(30,33$ e $\left.36^{\circ} \mathrm{C}\right)$, no fluxo da FM $\left(0,80,1,00\right.$ e $\left.1,20 \mathrm{~mL} \cdot \mathrm{min}^{-1}\right)$, na composição da fase orgânica $(45,0,47,0$ e 49,0\% ACN) e na adição de ácido fosfórico na FM aquosa $(0,05,0,10$ e 0,20\% - v/v) em água ultrapura na concentração de $100,0 \mu \mathrm{g} \cdot \mathrm{L}^{-1}$. Os resultados demonstraram a robustez da técnica, e os perfis dos cromatogramas são apresentados na Figura 4.
Em relação à variação do fluxo, não foi observada variação na intensidade dos picos dos compostos, apenas variações no $t_{R}$ dos analitos (Figura 4A), assim como na variação da porcentagem da fase orgânica (Figura 4B). Com base nos resultados, verificou-se que o método apresentou robustez com concentrações de ácido acético no intervalo de 0,05 a 2,0\% (Figura 4C), pois nessa faixa de concentração não ocorreram diferenças significativas nas resoluções cromatográficas. Sem a adição do ácido fosfórico, os compostos 2,4-D e 2,4,5-T não foram detectados por serem compostos ionizáveis e pouco retidos em fase reversa. Nesse caso, o ideal é trabalhar com pH abaixo do pKa da substância, condição obtida com adição do ácido fosfórico, pois o pH influencia na ionização de ácidos, aumentando a retenção em fase

\section{A}

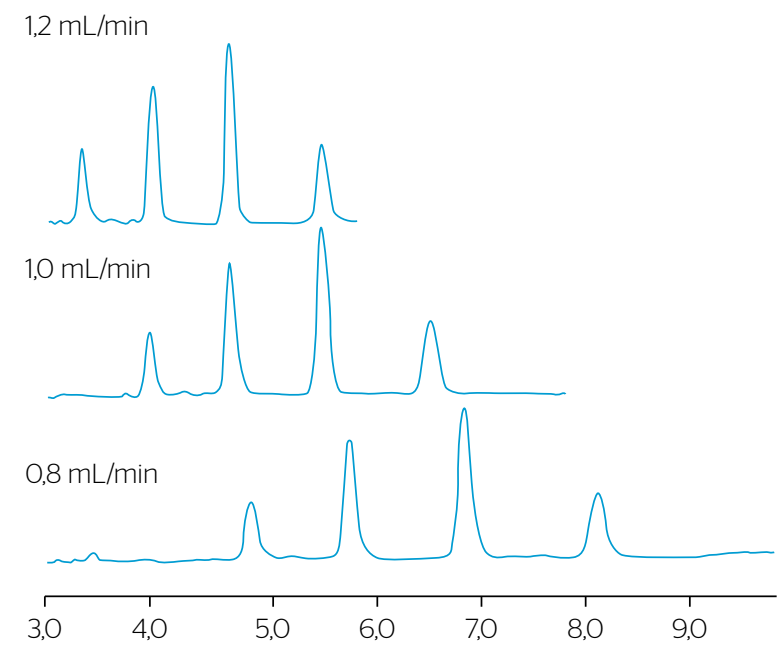

C

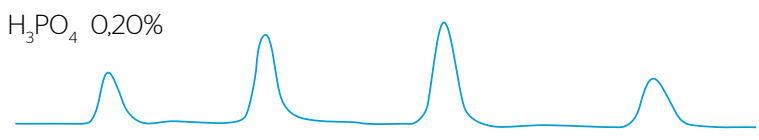

$\mathrm{H}_{3} \mathrm{PO}_{4} \quad 0,10 \%$

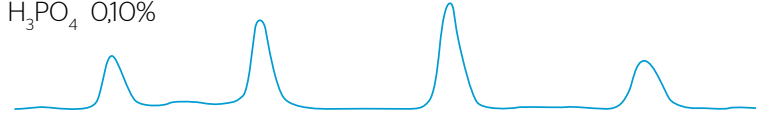

$\mathrm{H}_{3} \mathrm{PO}_{4} \quad 0,05 \%$

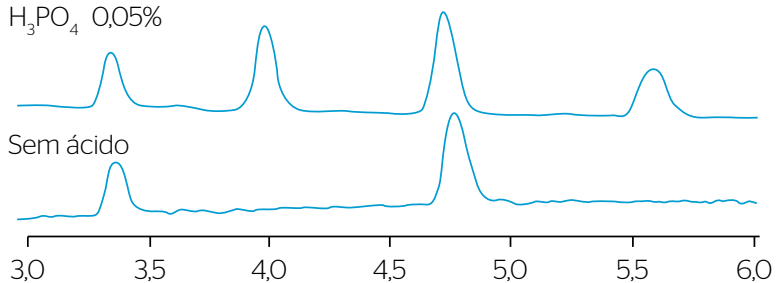

B
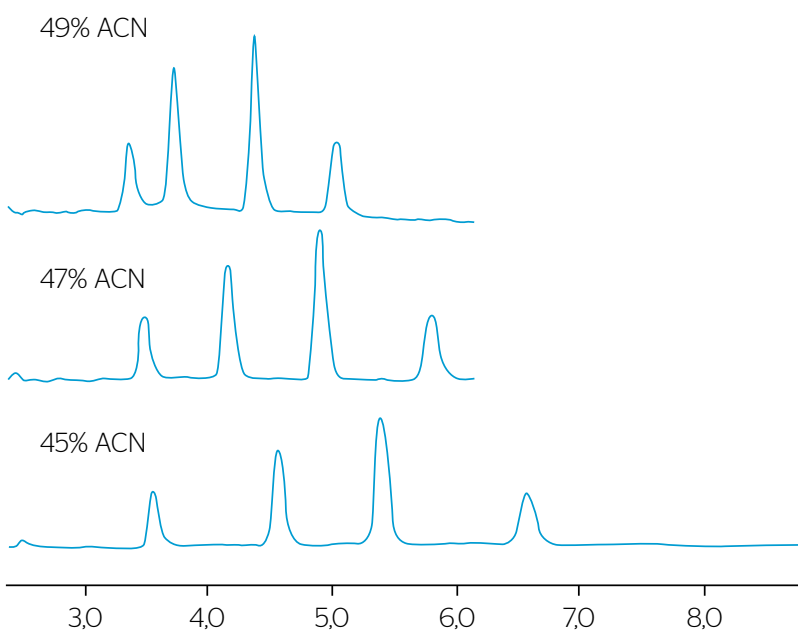

D

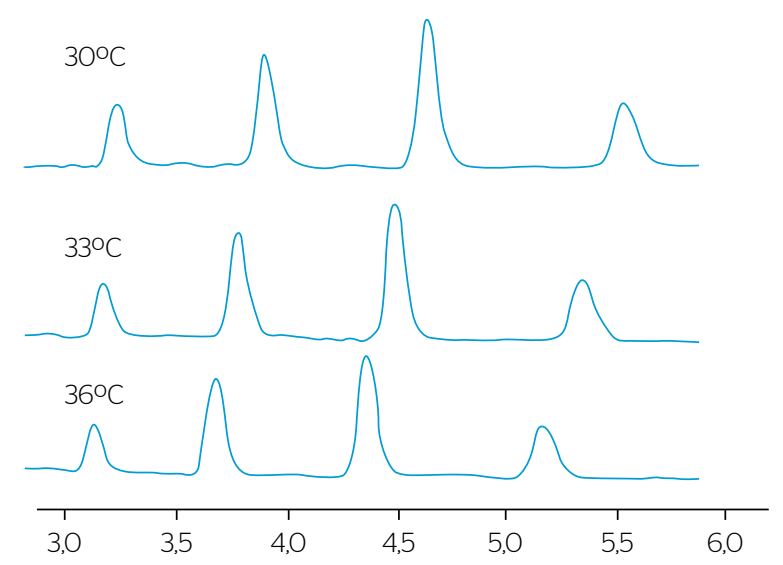

Figura 4 - Perfis cromatográficos referentes aos padrões de 2,4-D, 2,4-DCP e 2,4,5-T em água ultrapura na concentração de 100,0 $\mu$ g.L'1. (A) Diferentes fluxos; (B) diferentes concentrações de acetonitrila; (C) diferentes concentrações de ácido fosfórico adicionado à fase móvel aquosa $(\mathrm{v} / \mathrm{v})$; (D) diferentes temperaturas de acondicionamento da coluna. 
reversa (LANÇAS, 2009; BAGGIANI et al., 2000). A resolução cromatográfica obtida com a FM contendo $\mathrm{H}_{3} \mathrm{PO}_{4} 0,10 \%(\mathrm{pH} 2,0 \pm 0,10)$ foi definida como condição ótima para os demais ensaios cromatográficos. Em relação à variação da temperatura de acondicionamento da coluna, as resoluções cromatográficas entre os analitos não apresentaram diferenças, além de pequenas variações nos $t_{R}$ (Figura $4 D$ ), mostrando-se robusto.

A técnica cromatográfica se mostrou simples, rápida e satisfatória para a análise simultânea de 2,4-D, 2,4-DCP e 2,4,5-T em amostras ambientais de água, podendo ser aplicada no controle e no monitoramento de mananciais e ETAs.

\section{CONCLUSÕES}

O método de análise de agrotóxicos em água utilizando EFS e CLAE$\mathrm{DAD}$ demonstrou seletividade, linearidade $(\mathrm{r} \geq 0,997)$, precisão $(\mathrm{CV} \leq 12 \%)$ e exatidão $\geq 85 \%$ para separar e quantificar simultaneamente os compostos 2,4-D, 2,4-DCP e 2,4,5-T em água filtrada e água de manancial superficial. Os valores de LD entre 0,17 e 0,51 $\mu \mathrm{g} . \mathrm{L}^{-1}$ e de LQ de 1,0 $\mu \mathrm{g} . \mathrm{L}^{-1}$ atendem aos limites estabelecidos pela Portaria MS no 2.914/2011. Portanto, o método apresentado pode ser utilizado para a detecção e a quantificação concomitante de 2,4-D, 2,4-DCP e 2,4,5-T em águas de abastecimento público e no controle e no monitoramento ambiental, atendendo os limites estabelecidos nas legislações brasileiras.

\section{REFERÊNCIAS}

AGÊNCIA NACIONAL DE VIGILÂNCIA SANITÁRIA (ANVISA). (2O03) Resolução no 899, de 29 de maio de 2003. Determina a Publicação do "Guia para validação de métodos analíticos e bioanalíticos". Diário Oficial da União, Brasília.

ASSOCIATION OF OFFICIAL ANALYTICAL CHEMISTRY (AOAC). (1998) Peer verified method program: manual on policies and procedures. Arlington: AOAC. 35 p.

AULAKH, J.S.; MALIK, A.K.; KAUR, V.; SCHMITT-KOPPLIN, P. (2OO5) A Review on Solid Phase Micro Extraction - High Performance Liquid Chromatography (SPMEHPLC) Analysis of Pesticides. Critical Reviews in Analytical Chemistry, v. 35, n. 1, p. 71-85. https:// doi.org/10.1080/10408340590947952

AZEVEDO, D.A.; GERCHON, E.; REIS, E.O. (2004) Monitoring of Pesticides and Polycyclic Aromatic Hydrocarbons in Water from Paraiba do Sul River, Brazil. Journal of the Brazilian Chemical Society, v. 15, p. 299. http://dx.doi.org/10.1590/S0103-50532004000200021

BAGGIANI, C.; GIOVANNOLI, C:; ANFOSSI, L.; TOZZI, C. (2001) Molecularly imprinted solid-phase extraction sorbent for the cleanup of chlorinated phenoxyacids from aqueous samples. Journal of Chromatography A, v. 938, p. 35-44.

BAGGIANI, C.; GIRAUDI, G.; GIOVANNOLI, G.; TROTTA, F.; VANNI, A.J. (2000) Chromatographic characterization of molecularly imprinted polymers binding the herbicide 2,4,5-trichlorophenoxyacetic acid. Journal of Chromatography A, v. 883, p. 119-126.

BRASIL. Ministério da Saúde. (2011) Portaria n.o 2.914 de 12 de dezembro de 2011. Dispõe sobre os procedimentos de controle e de vigilância da qualidade da água para consumo humano e seu padrão de potabilidade. Diário Oficial da União, Brasília.

BRONDI, S.H.G.; LANÇAS, F.M. (2005) Development and validation of a multi-residue analytical methodology to determine the presence of selected pesticides in water through liquid chromatography. Journal of the Brazilian Chemical Society, v. 16, p. 650-653. http:// dx.doi.org/10.1590/S0103-50532005000400026
COMISSÃO DAS COMUNIDADES EUROPÉIAS (CCE). (2001) Estratégia comunitária em matéria de desreguladores endócrinos: substâncias suspeitas de interferir com os sistemas hormonais dos seres humanos e dos animais. Bruxelas: COM 706. 49 p.

D’ARCHIVIO, A.A.; FANELLI, M.F.; MAZZEO, P.; RUGGIERI, F. (2007) Comparison of different sorbents for multiresidue solid-phase extraction of 16 pesticides from groundwater coupled with highperformance liquid chromatography. Talanta, v. 71, p. 25-30. https:// doi.org/10.1016/j.talanta.2006.03.016

DE ARMAS, E.D.; MONTEIRO, R.T.R.; ANTUNES, P.M.; DOS SANTOS, M.A.P.F.; DE CAMARGO, P.B.; ABAKERLI, R.B. (2007) Diagnóstico espaço-temporal da ocorrência de herbicidas nas águas superficiais e sedimentos do rio Corumbataí e principais afluentes. Química Nova, v. 30, p. 1119-1127. http://dx.doi.org/10.1590/S010040422007000500013

DORES, E.F.G.C.; NAVICKIENE, S.; CUNHA, M.L.F.; CARBO, L.; RIBEIRO, M.L.; DE-LAMONICA-FREIRE, E.M. (2006) Multiresidue Determination of Herbicides in Environmental Waters from Primavera do Leste Region (Middle West of Brazil) by SPE-GC-NPD. Journal of the Brazilian Chemical Society, v. 17, p. 866-873. http:// dx.doi.org/10.1590/S0103-50532006000500008

FARIA, L.J.S. (2004) Avaliação de diferentes sorventes na extração em fase sólida de pesticidas em água. Desenvolvimento e validação de metodologia. Dissertação (Mestrado em Química) Universidade Estadual de Campinas, Campinas. 79 p.

FUKUYAMA, T.; TAJIMA, Y.; UEDA, H.; HAYASHI, K.; SHUTOH, Y.; HARADA, T.; KOSAKA, T. (2009) Allergic reaction induced by dermal and/or respiratory exposure to low-dose phenoxyacetic acid, organophosphorus, and carbamate pesticides. Toxicology, v. 261, p. 152-161. https://doi.org/10.1016/j.tox.2009.05.014

GOODMAN, J.E.; LOFTUS, C.T:ZU, K. (2017)2,4-Dichlorophenoxyacetic acid and non-Hodgkin's Iymphoma: results from the Agricultural Health Study and an updated meta-analysis. Annals of Epidemiology, v. 27, p. 290-292. https://doi.org/10.1016/j.annepidem.2017.01.008 
LANÇAS, F.M. (2009) Cromatografia Líquida Moderna: HPLC/CLAE. Campinas: Átomo. 382 p.

(2012) Disciplina: como desenvolver e otimizar um método em HPLC (teoria e prática). São Carlos: Instituto Internacional de Cromatografia.

LOOMIS, D.; GUYTON, K.; GROSSE, Y.; GHISSASI, F.E.; BOUVARD, V.; BENBRAHIM-TALLAA, L.; GUHA, N.; MATTOCK, H.; STRAIF, K.; AGENNCIA INTERNACIONAL DE PESQUISAS DO CÂNCER (IARC). (2015) Carcinogenicity of lindane, DDT, and 2,4-dichlorophenoxyacetic acid. The Lancet Oncology, v. 16, p. 891892. https://doi.org/10.1016/S1470-2045(15)00081-9

LOPES, M.N.T.; TOLEDO NETTO, P.; LOURENCETTI, C.; MARCHI, M.R.R.; RIBEIRO, M.L. (2011) Validação e aplicação de método para análise de pesticidas em água para consumo humano de Dourados (MS) por CLAE/UV e CG/DTE. Pesticidas: Revista de Ecotoxicologia e Meio Ambiente, v. 21, p. 103-116. http://dx.doi. org/10.5380/pes.v21iO.25914

MACEDO NETO, D.; FROEHNER, S.; MACHADO, K.S. (2012) Avaliação do transporte do ácido 2,4-diclorofenoxiacético através de um lisímetro. Química Nova, v. 35, p. 1809-1813. http://dx.doi.org/10.1590/ S0100-40422012000900020

MARCHESAN, E.; SARTORI, G.M.S.; AVILA, L.A.D.; MACHADO, S.L.D.O; ZANELLA, R.; PRIMEL, E.G.; MACEDO, V.R.M.; MARCHEZAN, M.G. (2O1O) Resíduos de agrotóxicos na água de rios da Depressão Central do Estado do Rio Grande do Sul, Brasil. Ciência Rural, Santa Maria, v. 40, p. 1053-1059. http://dx.doi.org/10.1590/S0103-84782010005000078

MCKNIGHT, U.S.; RASMUSSEN, J.J.; KRONVANG, B.; BINNING, P.J.; BJERG, P.L. (2015) Sources, occurrence and predicted aquatic impact of legacy and contemporary pesticides in streams. Environmental Pollution, v. 200, p. 64-76. https://doi.org/10.1016/j. envpol.2015.02.015

NAM, S.; JO, B.; YOON, Y.; ZOH, K.D. (2014) Occurrence and removal of selected micropollutants in a water treatment plant. Chemosphere, v. 95, p. 156-165. https://doi.org/10.1016/j.chemosphere.2013.08.055

NJOKU, V.O; HAMEED, B.H. (2011) Preparation and characterization of activated carbon from corncob by chemical activation with $\mathrm{H}_{3} \mathrm{PO}_{4}$ for 2,4-dichlorophenoxyacetic acid adsorption. Chemical Engineering Journal, v. 173, p. 391-399. http://dx.doi.org/10.1016\%2Fj.cej.2011.07.075

NJOKU, V.O.; ISLAM, A.; ASIF, M.; HAMEED, B.H. (2015) Adsorption of 2,4-dichlorophenoxyacetic acid by mesoporous activated carbon prepared from $\mathrm{H}_{3} \mathrm{PO}_{4}$ activated langsat empty fruit bunch. Journal of Environmental Management, v. 154, p. 138-144. https://doi. org/10.1016/j.jenvman.2015.02.002

OPEOLU, B.O.; FATOKI, O.S.; ODENDAAL, J. (2010) Development of a solid-phase extraction method followed by HPLC-UV detection for the determination of phenols in water. International Journal of the Physical Sciences, v. 5, p. 576-581.
PRIMEL, E.G.; ZANELLA, R.; KURZ, M.H.S.; GONÇALVES, F.F.; MACHADO, S.O.M.; MARCHEZAN, E. (2005) Poluição das águas por herbicidas utilizados no cultivo do arroz irrigado na região central do estado do Rio Grande do Sul, Brasil: predição teórica e monitoramento. Química Nova, v. 28, p. 605-609. http://dx.doi. org/10.1590/S0100-40422005000400010

RIBANI, M.; BOTTOLI, C.B.G.; COLLINS, C.H.; JARDIM, I.C.S.F.; MELO, L.F.C. (2004) Validação em métodos cromatográficos e eletroforéticos. Química Nova, v. 27, p. 771-780. http://dx.doi. org/10.1590/S0100-40422004000500017

RIBEIRO, A.C.A.; DORES, E.F.G.C.; AMORIM, R.S.S.; LOURENCETTI, C. (2013) Resíduos de pesticidas em águas superficiais de área de nascente do rio São Lourenço-MT: validação de método por extração em fase sólida e cromatografia líquida. Química Nova, v. 36 p. 284-290. http://dx.doi.org/10.1590/S0100-40422013000200015

RISSATO, S.R.; LIBÂNIO, M.; GIAFFERIS, G.P.; GERENUTTI, M. (2004) Determinação de pesticidas organoclorados em água de manancial, água potável e solo na região de Bauru (SP) Química Nova, v. 27, p. 739-743. http://dx.doi.org/10.1590/S010040422004000500012

SALVAGNI, J.; TERNUS, R.Z.; FUENTEFRIA, A.M. (2011) Assessment of the genotoxic impact of pesticides on farming communities in the country side of Santa Catarina State, Brazil. Genetics and Molecular Biology, v. 34, p. 122-126. http://dx.doi.org/10.1590/S141547572010005000104

SMITH, A.M.; SMITH, M.T.; LA MERRILL, M.A.; LIAW, J.; STEINMAUS, C. (2017) 2,4-dichlorophenoxyacetic acid (2,4-D) and risk of nonHodgkin lymphoma: a meta-analysis accounting for exposure levels. Annals of Epidemiology, v. 27, p. 281-289. https://doi. org/10.1016/j.annepidem.2017.03.003

SOUZA, F.L.; SÁEZ, C.; LANZA, M.R.V.; CAÑIZARES, P.; RODRIGO, M.A. (2017) Removal of chlorsulfuron and 2,4-D from spiked soil using reversible electrokinetic adsorption barriers. Separation and Purification Technology, v. 178, p. 147-153. http://dx.doi.org/10.1016/j. seppur.2017.01.030

TU, M.; HURD, C.; RANDALL, J.M. (2001) Weed control methods handbook: tools \& techniques for use in natural areas. Logan: Utah State University, U.S. Government Documents. 220 p.

WANG, Y.R.; CHU, W. (2011) Degradation of 2,4,5-trichlorophenoxyacetic acidby a novel Electro-Fe(II)/Oxone process using iron sheet as the sacrificial anode. Water Research, v. 45, p. 3883-3889. https://doi.org/10.1016/j.watres.2011.04.034

WOOD, R. (1999) How to validate analytical methods. Trends in Analytical Chemistry, v. 18, p. 624-632.

XI, Y.; MALLAVARAPU, M.; NAIDU, R. (2010) Adsorption of the herbicide 2,4-D on organo-palygorskite. Applied Clay Science, v. 49, p. 255-261. 